\title{
Unravelling an Extra Neutral Gauge Boson at the LHC Using Third Generation Fermions
}

\author{
Ross Diener ${ }^{1,2}$, Stephen Godfrey ${ }^{1,3 *}$ and Travis A. W. Martin ${ }^{1 \dagger}$ \\ ${ }^{1}$ Ottawa-Carleton Institute for Physics, Department of Physics, Carleton University, Ottawa, Canada K1S 5 B6 \\ 2 Perimeter Institute for Theoretical Physics, 31 Caroline St N, Waterloo, Canada N2L $2 Y 5$ \\ ${ }^{3}$ TRIUMF, 4004 Wesbrook Mall, Vancouver, Canada V6T $2 A 3$
}

(Dated: November 12, 2018)

\begin{abstract}
We study the potential to use measurements of the properties of extra neutral gauge bosons $\left(Z^{\prime}\right.$ 's) in $p p$ collisions at the Large Hadron Collider to unravel the underlying physics. We focus on the usefulness of third generation final states $(\tau, b, t)$ in distinguishing between models with non-universal $Z^{\prime}$-fermion couplings. We present an update of discovery limits of $Z^{\prime}$ 's including the 2010-2011 LHC run and include models with non-universal couplings. We show how ratios of $\sigma\left(p p \rightarrow Z^{\prime} \rightarrow t \bar{t}\right), \sigma\left(p p \rightarrow Z^{\prime} \rightarrow b \bar{b}\right)$, and $\sigma\left(p p \rightarrow Z^{\prime} \rightarrow \tau^{+} \tau^{-}\right)$to $\sigma\left(p p \rightarrow Z^{\prime} \rightarrow \mu^{+} \mu^{-}\right)$can be used to distinguish between models and measure parameters of the models. Of specific interest are models with preferential couplings such as models with generation dependent couplings. We also find that forward-backward asymmetry measurements with third generation fermions in the final state could provide important input to understanding the nature of the $Z^{\prime}$. Understanding detector resolution and efficiencies will be crucial for extracting results.

PACS numbers: 12.60.Cn, 12.15Ji, 14.70.Pw, 12.15.-y
\end{abstract}

\section{INTRODUCTION}

With the startup of the Large Hadron Collider (LHC), it is possible that direct experimental evidence of new physics will soon follow. Many theoretical models of physics beyond the Standard Model include a new colourless, spin-1, neutral gauge boson $\left(Z^{\prime}\right)[1-5]$. A kinematically accessible $Z^{\prime}$ would be one of the most distinctive signals at the LHC, and may potentially be one of the earliest discoveries. If a heavy neutral gauge boson were discovered, the immediate task would be to understand its origins by measuring its properties. This subject has been explored extensively and continues to be an important topic of on-going research [6 14].

The ATLAS [15] and CMS [16] experiments at the LHC expect to be able to identify $\tau$ leptons and $b$ - and $t$-quark jets in $p p$ collisions. In a recent letter, we suggested that third generation fermions can provide an important tool for discriminating between candidate models of $Z^{\prime}$ s $[7$. Along this vein, we found that such measurements can also be used to explore the gauge symmetry of some models of new physics that include a $Z^{\prime}$ with distinctive properties reflecting the mixing between the underlying gauge groups. These include models with fermion type dependent couplings (i.e., preference for quarks over leptons) [17-19], or generation dependent couplings (i.e., preference for third generation) 20 31]. The models with generation dependent couplings, such as extended technicolour 24 26 and topcolor assisted technicolour 27 31], are especially interesting as they take the heavy top quark mass as evidence for new physics that is con-

\footnotetext{
*Email: godfrey@physics.carleton.ca

†Email: tmartin@physics.carleton.ca
}

nected to the mechanism of electroweak symmetry breaking (EWSB). Thus, evidence for the violation of generation universality could give important hints to the mechanism of EWSB. For such models, standard analysis methods that rely on Drell-Yan processes with either electron or muon final states may fail to identify important details of the underlying physics.

In this paper, we look at a number of measurements involving third generation final states, expanding on our previous letter [7]. We start by exploring the discovery reach for the models we consider. However, our main focus is to consider the viability and usefulness of measurements involving third generation fermions in identifying the underlying theory of a newly discovered $Z^{\prime}$. The measurements we study are the ratio of the third generation quark cross sections to the muon cross section, the ratio of the tau cross section to the muon cross section, and the forward-backward asymmetry with bottom and top-quark final states.

We found that the ratios of the branching fraction of $\tau^{+} \tau^{-}, b \bar{b}$ and $t \bar{t}$ final states to the $\mu^{+} \mu^{-}$final state in $Z^{\prime}$ decays are especially useful for understanding models with preferential couplings and can be used to quantify the extent of the preference. We also found that $A_{F B}$ measurements with third generation quarks in the final state have the potential to provide $Z^{\prime}$-fermion coupling information. Several papers have been published in the past on related topics [32 40]. This paper takes into account recent information on the expected capability of the ATLAS [15] and CMS [16] detectors in addition to backgrounds.

We begin in Section II with a brief survey of models with non-universal couplings to $Z^{\prime}$ 's, followed in Section III with details of our calculations, focusing on issues of third generation fermion identification and Standard Model backgrounds. In Section IV we give our results, 
starting with an update of discovery limits for $Z$ 's from the models we consider in this paper and reflecting the updated LHC running plans. Our main focus, however, is exploring how one can use third generation fermions to learn about the underlying theory that gives rise to a $Z^{\prime}$. Finally, we summarize our main conclusions in Section V.

\section{MODELS}

New massive resonances are present in many models, including $\mathrm{KK}$ theories with finite size extra dimensions, string theories and theories with extended gauge sectors. In this paper we focus on models with extended gauge sectors. Some examples of these models are variations of Little Higgs Models (Littlest Higgs $(\mathrm{LH})$ with $\tan \phi_{H}=$ 1.0 [4]], Simplest Little Higgs (SLH) [42], Anomaly Free Simple Little Higgs (AFSLH) [43]), $E_{6}$ models $(\chi, \psi, \eta)$, Left Right Symmetric models (LRM, ALRM) $\left(g_{R}=g_{L}\right)$, and 3-3-1 models [44]. The details of the E6 and LR Symmetric models have been described elsewhere, so we refer the interested reader to the literature [1 5].

We single out a subset of models where spontaneous breaking to diagonal subgroups results in generators corresponding to gauge bosons that couple to either different generations (first vs third) or different types (quark vs lepton) of fermions with differing strengths. These models typically have a group structure of the form $S U(2) \times S U(2)$ or $U(1) \times U(1)$. Such models include the Ununified Standard Model [17], non-commuting extended technicolor [24], top-flavour [21 23], and topcolor assisted technicolor models [27]. These models can be distinguished using third generation fermions and we therefore give some relevant details of the models that are used in our analysis.

\section{A. Ununified Model (UUM)}

In the Ununified Model [17] the left handed quarks and leptons transform as doublets under their respective $S U(2)$ groups in $S U(2)_{q} \times S U(2)_{l} \times U(1)_{Y}$. Right handed fermions transform as singlets under both groups, and hypercharge assignments remain the same as in the SM.

After symmetry breaking, the mass eigenstates for the gauge bosons include a massless photon, and two massive $Z$ bosons. The light $Z^{0}$ boson deviates from SM couplings at order $\sin ^{2} \phi$, where $\phi$ is the mixing parameter between the two $S U(2)$ groups and is expected to be small. The heavy boson then couples to the lepton and quark sectors as:

$$
g_{Z^{\prime}}=g_{Z^{0}} c_{w}\left(\frac{T_{3 q}}{\tan \phi_{U U M}}-\tan \phi_{U U M} T_{3 l}\right) .
$$

Chivukula et al. [18, 19] found 95\% C.L. constraints on the value of $M_{Z^{\prime}}$ dependent on the mixing angle. In general, $M_{Z^{\prime}}>2 \mathrm{TeV}$ is required, with a stronger mass constraint for larger values of $\sin ^{2} \phi_{U U M}$. In our study, the use of $M_{Z^{\prime}}=1.5 \mathrm{TeV}$ is for comparison to other models without such constraints. Unless otherwise stated, we take $\sin \phi_{U U M}=0.5$, following the work of Chivukula et al. [18, 19].

\section{B. $S U(2)_{h} \times S U(2)_{l}$ - Extended Technicolor (ETC)}

In models of an extended $S U(2)_{h} \times S U(2)_{l}$ structure, fermion generations transform differently under each gauge group - the first two generations transform as a doublet under the $S U(2)_{l}$, while the third generation transforms as a doublet under the $S U(2)_{h}$. Subsequently, this extended gauge group is broken to its familiar diagonal subgroup, $S U(2)_{L}$, at some energy scale $\mu$. The electric charge operator is given by:

$$
Q=T_{3 l}+T_{3 h}+Y
$$

In diagonalizing the mass matrix for the neutral gauge bosons, a nearly $\mathrm{SM} Z^{0}$ arises with a $Z^{\prime}$ that couples only to left handed fermions. This $Z^{\prime}$ then has a greatly enhanced coupling strength to third generation fermions, with couplings given by:

$$
g_{L}=g_{Z^{0}} c_{w}\left(-\tan \phi_{E T C} T_{3 h}+\cot \phi_{E T C} T_{3 l}\right) .
$$

Unless otherwise stated, a value of $\sin \phi_{E T C}=0.9$ is used. Constraints found by Chivukula et al., 19, 25. require $M_{Z^{\prime}}>2 \mathrm{TeV}$ for this value of $\sin \phi_{E T C}$. Again, calculations are performed with lower values of the $M_{Z^{\prime}}$ for comparisons with other models.

Examples of these models include top-flavour models [21 23] and non-commuting extended technicolor [2426].

\section{C. $U(1)_{h} \times U(1)_{l}-$ Topcolor Assisted Technicolor (TC2)}

The large mass of the top quark has led to suggestions that the top quark is intrinsically related to the dynamics of electroweak symmetry breaking. Models based on this idea are often called Topcolor [45, 46], and contain a gauge structure that is generation dependent. It is assumed that the QCD gauge group and the hypercharge $U(1)$ arises from the breaking of a larger group as in $S U(3)_{1} \times S U(3)_{2} \times U(1)_{1} \times U(1)_{2} \rightarrow S U(3)_{c} \times U(1)_{Y}$ with a residual, high scale $S U(3)^{\prime} \times U(1)^{\prime}$. In this type of model, the $Z^{\prime}$ plays a role in the generation of the large top quark mass by providing a tilting mechanism for the top quark seesaw 35.

The third generation fermions transform under the $U(1)_{1}$ group, while the first two generation fermions transform under the $U(1)_{2}$ group. After symmetry breaking, the resulting $Z^{\prime}$ couples differently to the third generation fermions than it does to the first two generation 
fermions. The couplings of the SM fermions to the $Z^{\prime}$ are given by:

$$
\begin{gathered}
g_{L, R}^{3}=\frac{1}{2} g_{Z^{0}} s_{w} Y_{S M} \cot \phi_{T C 2} \\
g_{L, R}^{1,2}=\frac{1}{2} g_{Z^{0}} s_{w} Y_{S M} \tan \phi_{T C 2},
\end{gathered}
$$

where $Y_{S M}$ is the standard model hypercharge value. Unless otherwise stated, a value of $\sin \phi_{T C 2}=0.5$ is used.
Examples of these models also include top quark seesaw models [27-29] and flavour-universal TC2 [30, 31].

\section{CALCULATIONS}

For our calculations we use the leading order Drell-Yan cross section. While it can be found in the literature, we include it here for completeness [5, 47, 48]]:

$$
\frac{d \sigma}{d \cos \hat{\theta}}\left(p p \rightarrow Z^{\prime}, Z^{0}, \gamma \rightarrow f \bar{f}\right)=\sum_{q} \int d x_{a} d x_{b} f_{q}\left(x_{a}, Q^{2}\right) f_{\bar{q}}\left(x_{b}, Q^{2}\right) \frac{d \hat{\sigma}(\hat{\theta})}{d \cos \hat{\theta}}+f_{\bar{q}}\left(x_{a}, Q^{2}\right) f_{q}\left(x_{b}, Q^{2}\right) \frac{d \hat{\sigma}(\pi-\hat{\theta})}{d \cos \hat{\theta}}
$$

where $d \sigma / d \cos \theta$ is given by

$$
\frac{d \hat{\sigma}}{d \cos \hat{\theta}}\left(q \bar{q} \rightarrow Z^{\prime}, Z^{0}, \gamma \rightarrow f \bar{f}\right)=\frac{\pi \alpha_{e m}^{2} \beta_{f}}{8 c_{W}^{4} s_{W}^{4} \hat{s}}\left\{\left(1+\beta_{f}^{2} \cos ^{2} \hat{\theta}\right) S_{q}+2 \beta_{f} \cos \hat{\theta} A_{q}+S_{q}^{\prime}\right\}
$$

and

$$
\begin{array}{r}
S_{q}, A_{q}=\sum_{i, j=\gamma, Z, Z^{\prime}}\left(\frac{\hat{s}}{\hat{s}-M_{i}^{2}-i \Gamma_{i} M_{i}}\right)\left(\frac{\hat{s}}{\hat{s}-M_{j}^{2}+i \Gamma_{j} M_{j}}\right) \times\left(R_{f}^{i} R_{f}^{j} \pm L_{f}^{i} L_{f}^{j}\right)\left(R_{q}^{i} R_{q}^{j} \pm L_{q}^{i} L_{q}^{j}\right) \\
S_{q}^{\prime}=\sum_{i, j=\gamma, Z, Z^{\prime}}\left(\frac{4 m_{f}^{2}}{\hat{s}}\right)\left(\frac{\hat{s}}{\hat{s}-M_{i}^{2}-i \Gamma_{i} M_{i}}\right)\left(\frac{\hat{s}}{\hat{s}-M_{j}^{2}+i \Gamma_{j} M_{j}}\right) \times\left(R_{f}^{i} L_{f}^{j}+L_{f}^{i} R_{f}^{j}\right)\left(R_{q}^{i} R_{q}^{j}+L_{q}^{i} L_{q}^{j}\right) .
\end{array}
$$

In Eq. (6) through (9), $M_{i}$ and $\Gamma_{i}$ are the masses and widths of the photon, $\mathrm{SM} Z^{0}$, and $Z^{\prime} ; L_{f}^{i}, R_{f}^{i}$ are the left and right handed couplings of the gauge bosons to fermion species $f ; m_{f}$ is the mass of the final state fermion; $f_{q, \bar{q}}\left(x, Q^{2}\right)$ are the parton distribution functions (pdf's); $x_{a}$ and $x_{b}$ are the momentum fractions of the partons; $Q^{2}$ is the scale at which the parton distribution functions are evaluated, which we take to be $\hat{s}$ - the square of the parton centre-of-mass energy; $\hat{\theta}$ is the centre-of-mass scattering angle; and $\beta_{f}=$ $\sqrt{1-4 m_{f}^{2} / \hat{s}}$. In our calculations we used $\alpha=1 / 127.9$, $\sin ^{2} \theta_{w}=0.231, M_{Z}=91.188 \mathrm{GeV}, \Gamma_{Z}=2.495 \mathrm{GeV}$ and $m_{t}=171.2 \mathrm{GeV}$ [49], and for the pdf's we used set CTEQ6M [50]. We calculated the $Z^{\prime}$ width including only $Z^{\text {/prime }}$ decays to SM fermions, and neglected $Z-Z^{\prime}$ mixing and decays to pairs of $W$ and $Z^{0}$ bosons, as well as decays to heavy, fourth generation fermions.

The total cross section is proportional to the $S_{q}$ and $S_{q}^{\prime}$ terms (with summed couplings), which are dependent on symmetric combinations of the $Z^{\prime}$-fermion couplings. The antisymmetric combinations of the $Z^{\prime}$ fermion couplings in the $A_{q}$ term contributes to the forward-backward asymmetry, $A_{F B}$. Thus, measur- ing the production cross section and forward-backward asymmetry of the decay of a $Z^{\prime}$ to third generation fermions will give complementary information about the $Z^{\prime}$ couplings to fermions - information that will be crucial for disentangling the underlying theory. The mass dependent coefficient for $S_{q}^{\prime}$ is only relevant for top decays when the mass of the $Z^{\prime}$ is relatively small. The effect is $O(10 \%)$ for a $1 \mathrm{TeV} Z^{\prime}$, dropping to $O(1 \%)$ for a $2 \mathrm{TeV} Z^{\prime}$, for top quark final states.

In our calculations, we include a K-factor [51] in the cross section to account for NLO QCD corrections and neglect NNLO as the uncertainties in parton distributions dominate over such small effects [52, 53]. We also included QCD [54 56] and EW [57 59] corrections in the width, with weak corrections having only a very minor effect.

The formula given in Eq. [6 was used to calculate the cross sections and distributions in this paper. The phase space integrals were performed using Monte-Carlo integration methods with weighted events. QCD backgrounds are calculated using a combination of the WHiZaRD (with O'MEGA matrix elements) [6062] and MADGRAPH [63] Monte-Carlo event generators and compared with our own code following Barger and 
Phillips [64]. All three background calculations agreed.

\section{IDENTIFICATION AND BACKGROUNDS}

The challenge to using third generation fermions in LHC events is to first identify the third generation fermions and then to identify the signal events buried in the large Standard Model QCD backgrounds. The backgrounds take two forms. The first is the SM production of third generation quarks which we will discuss below. The second is the misidentification of the large QCD light jet backgrounds as heavy fermions. Identification of third generation fermions and misidentification of light jet backgrounds are not unrelated. The rejection of light jet backgrounds is crucial to making accurate measurements of heavy quark final states. However, higher rejection typically results in lower identification efficiencies, and correspondingly lower statistics. There is therefore a tradeoff between high identification efficiency and suppression of the reducible background.

There has been recent activity on this subject with a number of papers appearing on the topic of the identification of top jets, specifically with techniques for highly boosted jets [36, 65 67]. In addition, ATLAS has released updated information on the expected capabilities of the detector for the tagging of leptons and bottom quarks [15]. We use this information to estimate the efficiency and rejection rates needed to analyze the data. It should be noted that some estimates are given for tagging lower energy events and we do not evaluate the validity of extending or extrapolating to higher energies. Better understanding of the ATLAS and CMS detectors will ultimately give more reliable values. Finally, there are non-QCD backgrounds such as $W+$ jets, $(W b+W \bar{b})$, and $W b \bar{b}+j e t s$. However, studies have shown that these can be controlled by constraints on cluster transverse mass and the invariant mass of jets [36] so we will not consider them further.

In the following subsections we summarize the identification efficiencies and fake rejection rates for the third generation fermions that we use in our analysis.

\section{A. Muon}

Muons are the most distinct of all tagged signatures for both ATLAS and CMS 68]. Recent studies by the ATLAS Muon Working Group [15] show a single muon identification efficiency greater than $95 \%$ for $p_{T}>30 \mathrm{GeV}$. These values vary over pseudorapidity and energy; for the purpose of this analysis, we use an efficiency of $\epsilon_{\mu}=96 \%$ for single muon identification and consider only Drell-Yan backgrounds.

\section{B. Tau Lepton}

There are three possible decay modes for a $\tau^{+} \tau^{-}$ event: purely leptonic $\left(\tau^{+} \tau^{-} \rightarrow \nu_{\tau} \bar{\nu}_{\tau} l^{+} \nu l^{-} \bar{\nu}, 12.4 \%\right.$ of total events), semi-leptonic events $\left(\tau^{+} \tau^{-} \rightarrow \nu_{\tau} \bar{\nu}_{\tau} l \nu+\right.$ jets, $45.6 \%$ of total events), and purely hadronic events $\left(\tau^{+} \tau^{-} \rightarrow \nu_{\tau} \bar{\nu}_{\tau}+j e t s, 42 \%\right.$ of total events $)$. Of the hadronic decays, $\approx 77 \%$ have one charged pion track (one-prong) and $\approx 23 \%$ have three charged pion tracks (three-prong). In our analysis, we consider the results from the purely hadronic mode, which suffers from a large reducible dijet background, as the leptonic modes have a larger missing energy component. It has been shown that a $\tau^{+} \tau^{-}$invariant mass distribution can be reconstructed in these modes even though the $\tau$ decays produce missing energy [69, 70] .

In order to reduce the dijet background, it will be necessary to implement tight tagging methods. In particular, ATLAS 15] estimates that it may be possible to achieve a rejection rate of $10^{3}$ and an efficiency of $20 \%$ for 3 prong decays with $E_{T}>100 \mathrm{GeV}$, and a rejection rate of $10^{3}$ and an efficiency of $50 \%$ for 1 prong decays with $E_{T}>100 \mathrm{GeV}$. This amounts to an overall rejection rate of $5 \times 10^{5}$ with $\approx 20 \%$ of all hadronic $(42 \%) \tau^{+} \tau^{-}$ events passing the selection criteria. The number of useful $\tau^{+} \tau^{-}$pairs can potentially be increased by including the leptonic and semileptonic modes.

\section{Bottom}

According to the current ATLAS algorithms [15], a jet is tagged as a $b$-jet if a $b$ - quark is found with $p_{T}>5 \mathrm{GeV}$ within $\Delta R=0.3$ around the centroid of the jet. Further improvements are made to this tag by including either the identification of a secondary vertex or else reconstructing the invariant mass of the jet. In an analysis of $b$-jets arising from $W H$ and $t \bar{t}$, a jet rejection of about $2 \times 10^{2}$ was found for a $b$-jet efficiency of $60 \%$ - or $36 \%$ for a $b \bar{b}$ pair, with a light jet rejection of $4 \times 10^{4}$. Tighter tagging could be employed to improve the rejection rate if the light jet background dominates the observed events.

\section{Top}

A complete analysis of physics events involving top quarks would require an analysis of the decay products to calculate an overall efficiency. This is beyond the scope of this paper and we instead use previous studies to estimate an overall efficiency [36, 65, 71 73 ].

We considered both the semi-leptonic decay mode $\left(t \bar{t} \rightarrow\left(l \nu_{l}\right)(j j) b \bar{b}\right.$ where $\left.l=\mu, e\right)$ with an overall $30 \%$ branching ratio, and fully hadronic modes $(t \bar{t} \rightarrow$ $(j j)(j j) b \bar{b})$ with an overall branching ratio of $46 \%$.

Semi-leptonic modes present the best signal-tobackground ratio, with the dominant fake background being $W+n j$. An analysis of KK modes for the LHC 
by Agashe et al., 74 found an overall efficiency of $\approx 1 \%$, after taking into account branching fraction, cuts and $b$-tagging efficiency. Their analysis included a $20 \%$ efficiency for the $b$-tag. They found the reducible background to be small compared to the irreducible QCD background.

In a separate analysis, Kaplan et al. [65] considered a method of top tagging for high $p_{T}$ hadronic top decays that suggests better efficiencies and does not incorporate a $b$-tag. Their analysis using Pythia and a detailed systematic examination of top decays suggests a tagging efficiency of approximately $35 \%$ to $45 \%$ for top jets with transverse momentum between 600 and $1400 \mathrm{GeV}$. For fully hadronic $t \bar{t}$ events, this technique could result in an efficiency $>10 \%$ for $\approx 46 \%$ of events, and a rejection against light jets $>10^{4}$. Very specifically, the dijet reducible background is shown to be reduced to the same level as the QCD $t \bar{t}$ background, where a resonant peak may be visible.

Almeida et al. 73] found that using only a cut on the jet mass resulted in a single top jet identification efficiency of between $34 \%$ and $58 \%$ with a rejection factor of around 30. By applying further cuts on the jet structure, they were able to achieve a rejection factor of about 5000 for a top identification efficiency of $\approx 21 \%$. This is in general agreement with the Kaplan study in that reasonable top jet tagging efficiencies can be expected at the LHC without significant light jet contamination.

In our analysis, we assume the method used by Kaplan et al. and employ an efficiency of $16 \%$ (40\% for each top jet - the midpoint of the expected range) for the fully hadronic decay mode, with a rejection of dijets by a factor of $10^{4}$.

\section{E. Tagging Summary}

We summarize the tagging efficiencies we use in Table [1. For $\tau$ and $t$ decays, we only consider the fully hadronic decay channels. The efficiencies are used to determine the statistical uncertainties we give in our results. For ratios of cross sections we assume that experimentalists have properly taken into account efficiencies to extract the appropriate cross section.

TABLE I: Summary of the overall efficiencies used for estimating the number of events observed of the given fermion species. The overall efficiency is the tagging efficiency for the observed fermion decay mode times the BR to that final state.

\begin{tabular}{l|c|c} 
Channel & Overall $\epsilon_{f}$ & Jet Rejection $\epsilon_{j}$ \\
\hline$Z^{\prime} \rightarrow \mu^{+} \mu^{-}$ & 0.92 & $\mathrm{n} / \mathrm{a}$ \\
$Z^{\prime} \rightarrow \tau^{+} \tau^{-}$ & 0.08 & $2.0 \times 10^{-6}$ \\
$Z^{\prime} \rightarrow b \bar{b}$ & 0.36 & $2.5 \times 10^{-5}$ \\
$Z^{\prime} \rightarrow t \bar{t}$ & 0.075 & $1.0 \times 10^{-4}$
\end{tabular}

\section{F. Kinematic Cuts}

The values for the fake rates and efficiencies assume the ideal case of perfect alignment within the inner detector and no pile-up. Since tracking is only available within $|\eta|<2.5$, we do not include events in which one or both fermion tracks lie outside this region, and, unless otherwise stated, we require a minimum $p_{T}>20 \mathrm{GeV}$ as needed for flavour tagging. Figure 1 (a) shows the invariant mass distribution in the $b \bar{b}$ final state for several models, the QCD $b \bar{b}$ backgrounds, and the light dijet backgrounds.

For hadronic decays of $\tau, b$ and $t$ fermions, we found that a $p_{T}>0.3 M_{Z^{\prime}}$ cut on the reconstructed momentum of the hadronic jets effectively reduced both the irreducible and dijet backgrounds as compared to the signal. The events from the decay of a $Z^{\prime}$ tend towards a harder $p_{T}$ distribution than the QCD backgrounds so that a larger percentage of $Z^{\prime}$ events pass this cut than the QCD backgrounds. Figure 1 (b) shows the signal for several $Z^{\prime}$ models, QCD $b \bar{b}$ background, and the light dijet background after applying this strong $p_{T}$ cut. This cut was also applied to muon final states when calculating ratios of cross sections, for consistency. For determination of the discovery limits we only apply a cut of $p_{T}>20 \mathrm{GeV}$ to the final state muons.

Fig. 1 (c) shows the reduction of the light dijet background after taking into account detector efficiencies and fake rates. Figure 1 (c) shows that the application of appropriate flavour tagging algorithms reduces both the QCD $b \bar{b}$ and the light dijet backgrounds to a level where a meaningful measurement should be possible.

As a final kinematic cut, we include only events within an appropriate invariant mass window around the resonance mass to improve the signal to background. Unless otherwise stated, we take this to be $\left|M_{Z / \text { prime }}-M_{f \bar{f}}\right|<$ $2.5 \Gamma_{Z / \text { prime. }}$ This restricts the background events to the kinematic region directly under the resonance peak. However, this introduces an additional experimental uncertainty due to detector resolution, which smears out the resonance peak, effectively reducing the number of signal events in the peak. Because we use ratios of cross section measurements into $b \bar{b}, t \bar{t}, \mu^{+} \mu^{-}$, and $\tau^{+} \tau^{-}$final states, which have different energy resolutions, the numerical value of the observables will shift. Thus, it will be important to understand detector resolution to accurately extract the underlying cross sections and coupling dependence. In addition, the reduction of the measured signal compared to background will increase the experimental errors.

To properly account for detector resolution requires a realistic, detailed simulation for the specific particle identification algorithms being used. As discussed above, this subject is evolving rapidly. In addition, experimentalists are constantly improving their understanding of the energy calibration of the LHC detectors. To gauge the importance of detector resolution, we use estimates from recent detector studies and include them by ap- 

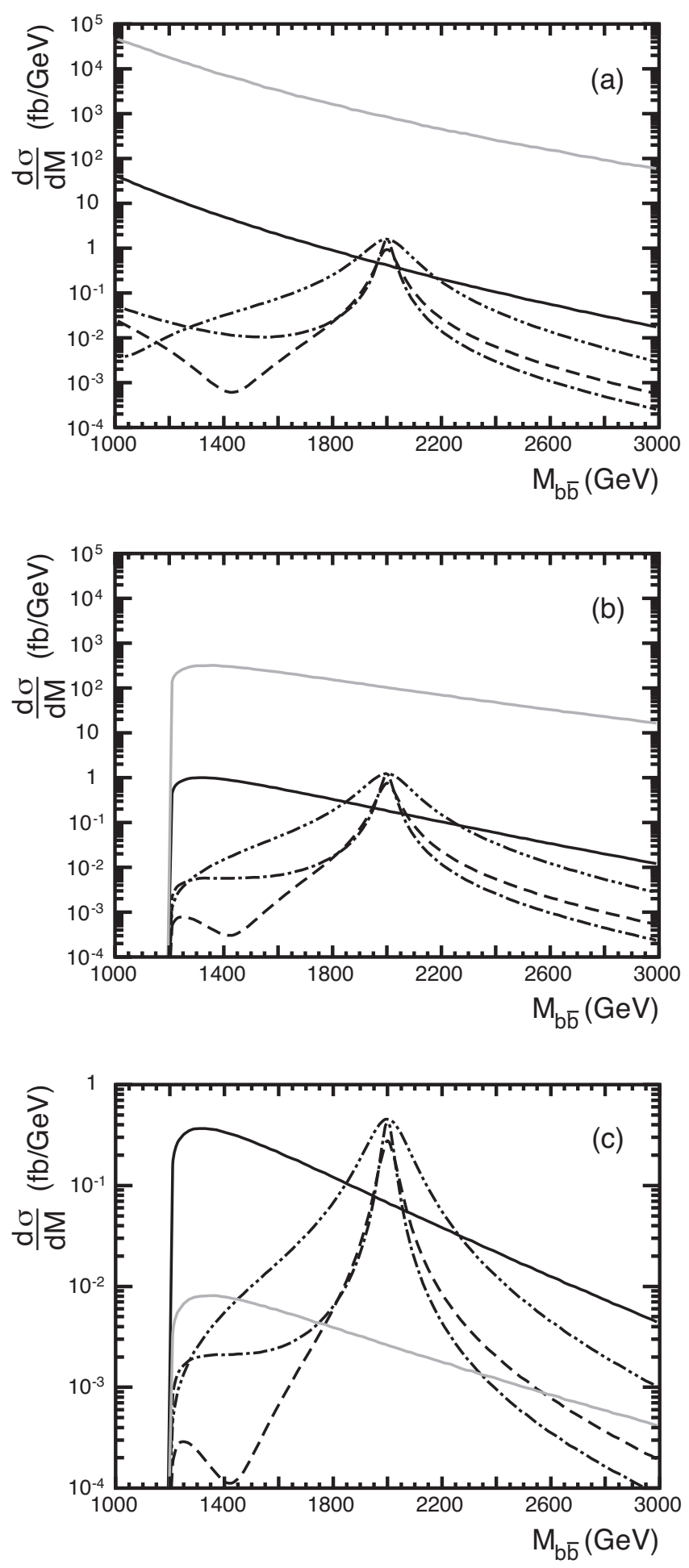

FIG. 1: $\quad b \bar{b}$ invariant mass distributions for QCD $b \bar{b}$ background (solid, dark), light dijet background (solid, grey), and a $Z^{\prime}$ with $M_{Z^{\prime}}=2.0 \mathrm{TeV}$ for the LRM (dot-dash), UUM (dot-dot-dash), and LH (dashed) models. (a) Only includes detector acceptance cuts of $p_{T}>20 \mathrm{GeV}$ and $|\eta|<2.5$. (b) Also includes $p_{T}>0.3 M_{Z^{\prime}}$ cut as described in the text but before including tagging efficiencies. (c) Includes the application of cuts, tagging efficiencies, and fake rejection rates given in Table I plying Gaussian smearing to the final state momentum. Studies by the ATLAS collaboration expect between $3 \%$ and $5 \%$ energy resolution for TeV scale hadronic jets 15 . (although the current ATLAS jet calibration gives $5 \%$ resolution [75]). This results in a resolution of $\sim 3.5 \%$ for $M_{b \bar{b}}$. This broadens the resonance, reducing the number of measured signal events within the same invariant mass window. Signal significance may therefore be significantly reduced for narrow resonances.

To see how detector resolution affects measurements, we include results for the ideal case of no smearing and for a more realistic case of $5 \%$ on $b$ and $t$ final states and $3 \%$ on muon final states. For the $b \bar{b}$ case we found that for the chosen mass window, as expected, detector resolution has the greatest effect for the narrowest resonances (e.g. the $Z_{\psi}^{\prime}$ ) with virtually no effect on the broadest states (e.g. the $Z_{U U M}^{\prime}$ ).

The situation for the $t \bar{t}$ final state is less conclusive for the following reason. A recent ATLAS study 76 gives a resolution of $4.6 \%$ for the $t \bar{t}$ invariant mass distribution of a narrow resonance. Using the same procedure used to gauge the importance of detector resolution for the $b \bar{b}$ channel we find that a usable signal can be measured in the $t \bar{t}$ channel for all models considered in this paper except for possibly $Z_{\eta}$ and $Z_{\psi}$. This is consistent with the findings of Barger, Han and Walker 71]. However, another ATLAS study [77] gives a resolution of $9-10 \%$ for a $1 \mathrm{TeV} Z^{\text {/prime }}$ with width $\Gamma_{Z / \text { prime }} / M_{Z / \text { prime }}=$ $3.3 \%$. The range of these expectations demonstrates the difficulty in trying to predict the detector resolution for these measurements.

Reconstruction of $\tau^{+} \tau^{-}$final states are complicated due to the missing energy from the neutrinos in the $\tau$ decays. LHC studies of $\tau^{+} \tau^{-}$final states with respect to Higgs searches have found a resolution on the reconstructed Higgs mass of $M_{H} \sim 10 \%[76,78,79]$. We do not incorporate this mass resolution in our results, but will refer to it when discussing our results for $\tau^{+} \tau^{-}$final states.

Thus, while we do estimate the effects that detector energy resolution will have on the precision of our measurements, past experience shows that the experimentalists eventually exceed initial expectations. In addition to this, the reduced signal to background caused by detector resolution can be mitigated to some extent by higher luminosities and better identification efficiencies, which would improve the statistics.

\section{RESULTS}

\section{A. Discovery Limits}

Leptonic final states offer the cleanest channel for the discovery of extra neutral gauge bosons due to the low backgrounds and clean identification [80 84]. A very small number of dilepton events clustered in one or two bins of the invariant mass distribution would be taken 


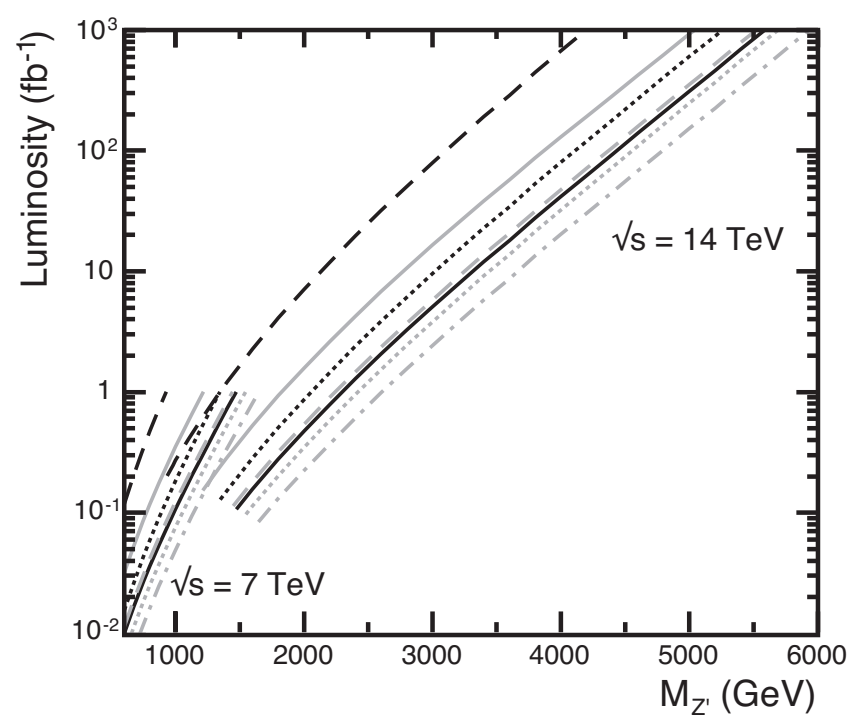

FIG. 2: Luminosity required for $Z^{\prime}$ discovery as a function of $M_{Z^{\prime}}$ based on the observation of $5 \mu^{+} \mu^{-}$events within the invariant mass window described in the text. The sets of curves are for an LHC centre of mass energy of $\sqrt{s}=$ $7 \mathrm{TeV}$ and $\sqrt{s}=14 \mathrm{TeV}$. ¿From left to right, the models are ETC (dashed, dark), TC2 (solid, grey), UUM (dotted, dark), AFSLH (dashed, grey), SLH (solid, dark), SSM (dotted, grey) and LH (dot-dash, grey).

as an obvious signal for new physics. To quantify this we consider two opposite sign leptons and impose kinematic cuts of $\left|\eta_{l}\right|<2.5$ and $p_{T_{l}}>20 \mathrm{GeV}$ to reflect detector acceptance. The criteria used was 5 events in the $\mu^{+} \mu^{-}$channel with a signal-over-background of at least 5 in an invariant mass window within \pm 1 bins of the resonance peak with the bin size as defined by $\Delta M=24\left(0.625 M+M^{2}+0.0056\right)^{1 / 2} \mathrm{GeV}$ where $M$ is given in $\mathrm{TeV}$ [85].

The integrated luminosity required to discover a $Z^{\prime}$ of a given mass in the dimuon channel is shown in Fig. 2. We show curves for $p p$ collisions with $\sqrt{s}=7 \mathrm{TeV}$ corresponding to the 2010-2011 LHC run and for $\sqrt{s}=14 \mathrm{TeV}$ which corresponds to the LHC design energy.

In Fig. 3 we show discovery limits for the various models for several LHC benchmark energies and luminosities and compare them to discovery limits for the Fermilab $p \bar{p}$ collider. For the Tevatron we assume two cases; $1.3 \mathrm{fb}^{-1}$ of integrated luminosity, as in Ref. [86], and $8 \mathrm{fb}^{-1}$ to estimate the reach for the full expected luminosity. We used similar detector acceptance and cuts as in Ref. [86]: we impose a kinematic cut of $p_{T}>25 \mathrm{GeV}$ and consider events within two regions of pseudorapidity - where both leptons satisfy $|\eta|<1.1$, and where one lepton satisfies $|\eta|<1.1$ and the other satisfies $1.2<|\eta|<2.0$. As well, we consider only events within an invariant mass window of $\left|M_{Z^{\prime}}-M_{l^{+} l^{-}}\right|= \pm 10 \% M_{Z^{\prime}}$. We use the discovery criteria of 5 observed dilepton events as with the

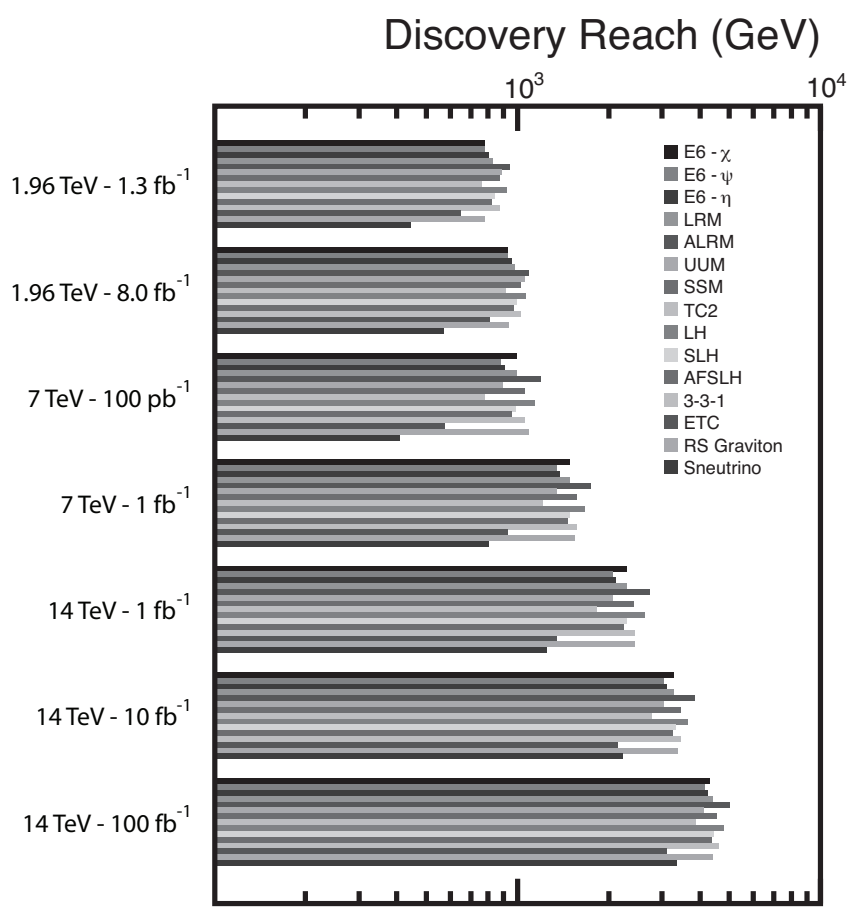

FIG. 3: Discovery reach at benchmark luminosities for Tevatron and LHC (both early and design) energies.

LHC study. We note that this differs from that used by the CDF collaboration to obtain the current direct limits in Refs. 86] and [87]. Figure 3 shows that the 2010-2011 LHC run will be able to roughly double the discovery reach of the Tevatron.

In addition, we considered the possibility that a $Z^{\prime}$ with a preferential coupling to third generation fermions may be discovered first in the $\tau^{+} \tau^{-}$final state. For the $\tau$ hadronic decay mode, none of the models we considered would be first visible in the $\tau$ final state for a value of mixing angle, $\phi$, that would give rise to a narrow resonance. At extreme values of the mixing angles in models without generation universality, the $Z^{\prime}$ begins to decouple from the first two generation fermions, reducing the $q \bar{q}-Z^{\prime}$ coupling, and hence the production of the $Z^{\prime}$. For these models, a $Z^{\prime}$ that would be first observed in the $\tau^{+} \tau^{-}$final state would have a width larger than $10 \%$ of the $Z^{\prime}$ mass, resulting in a very broad peak, unlikely to be observed. However, as described by Holdom [70], if other $\tau$ decay modes could be used, it may be possible to first observe a $Z^{\prime}$ in the $\tau^{+} \tau^{-}$final state for some regions of parameter space.

Constraints from electroweak precision data are more stringent than the discovery limits from the Tevatron for some models, as seen in Refs. 19, 88, 89]. However, it is clear that the $7 \mathrm{TeV}$ LHC run will improve the limits for models with universal couplings, and the full $14 \mathrm{TeV}$ run should improve the limits for non-universal models for reasonable values of the mixing angle, $\phi$. 


\section{B. Model Discrimination using $t \bar{t}$ and $b \bar{b}$ to $\mu^{+} \mu^{-}$ Production Ratios}

The primary goal of this paper is to explore the use of third generation fermions to distinguish between models of extra neutral gauge bosons. We start with ratios of $t \bar{t}$ and $b \bar{b}$ to $\mu^{+} \mu^{-}$cross sections, and expand on our previous study [7]. We are particularly interested in models with non-universal couplings - specifically, the UUM, ETC and TC2 models - as the $R_{t / \mu}$ and $R_{b / \mu}$ ratios defined below produce results that are quite distinctive from the models considered in Ref. 7].

$R_{t / \mu}$ and $R_{b / \mu}$ are defined by:

$$
\begin{aligned}
R_{t / \mu} & \equiv \frac{\sigma\left(p p \rightarrow Z^{\prime} \rightarrow t \bar{t}\right)}{\sigma\left(p p \rightarrow Z^{\prime} \rightarrow \mu^{+} \mu^{-}\right)} \approx \frac{3 K_{t}\left(L_{t}^{2}+R_{t}^{2}\right)}{\left(L_{\mu}^{2}+R_{\mu}^{2}\right)} \\
R_{b / \mu} & \equiv \frac{\sigma\left(p p \rightarrow Z^{\prime} \rightarrow b \bar{b}\right)}{\sigma\left(p p \rightarrow Z^{\prime} \rightarrow \mu^{+} \mu^{-}\right)} \approx \frac{3 K_{b}\left(L_{b}^{2}+R_{b}^{2}\right)}{\left(L_{\mu}^{2}+R_{\mu}^{2}\right)}
\end{aligned}
$$

where $L_{f}$ and $R_{f}$ are the left and right handed fermion couplings to the $Z^{\prime}$ and the $K$ factors incorporate the QCD and QED NLO correction factors [51, 54]. The use of the $R_{t / \mu}$ and $R_{b / \mu}$ ratios has the benefit of reducing the contributions from uncertainties in the parton distribution functions as the initial state $q \bar{q}$ couplings and pdf's cancel in the ratio.

To obtain our results, we assumed that a $Z^{\prime}$ has been discovered and its mass and width measured $[80,83,90$, 91] such that the appropriate $M_{Q \bar{Q}}$ and $p_{T}$ cuts described above can be applied. We calculated the expected number of events and statistical errors for signal plus background for a given integrated luminosity and particle identification efficiencies, $\epsilon_{\mu^{+} \mu^{-}}, \epsilon_{b \bar{b}}$, and $\epsilon_{t \bar{t}}$ from Table I. The expected number of SM QCD background events were subtracted from the total events to give the predicted number of signal events. We did not include systematic uncertainties arising from uncertainties in the luminosity and identification efficiencies.

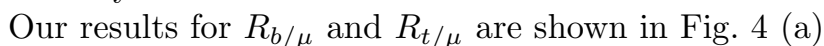
and (b), where Fig. 4 (b) expands the view to show the distinctive measurements that would be observed for models with preferential couplings. We show $1 \sigma$ statistical errors based on an integrated luminosity of $L=100 \mathrm{fb}^{-1}$. The measurements for models with preferential couplings and mixing parameters that result in large couplings for third generation quarks are quite distinctive, making this a possible hallmark measurement for these models.

In Fig. 5 we show $R_{b / \mu}$ and $R_{t / \mu}$ including a detector resolution of $5 \%$ for $b$ and $t$ final states, and $3 \%$ for muons. Comparing Fig. [5 with Fig. 4 it is clear that the effect of resolution is small for broad models such as ETC, TC2 and UUM. On the other hand, the effect is much more significant for the narrow models such as the E6, SLH, and AFSLH models, affecting both the values and expected statistical uncertainty. There are two approaches to account for resolution effects in these
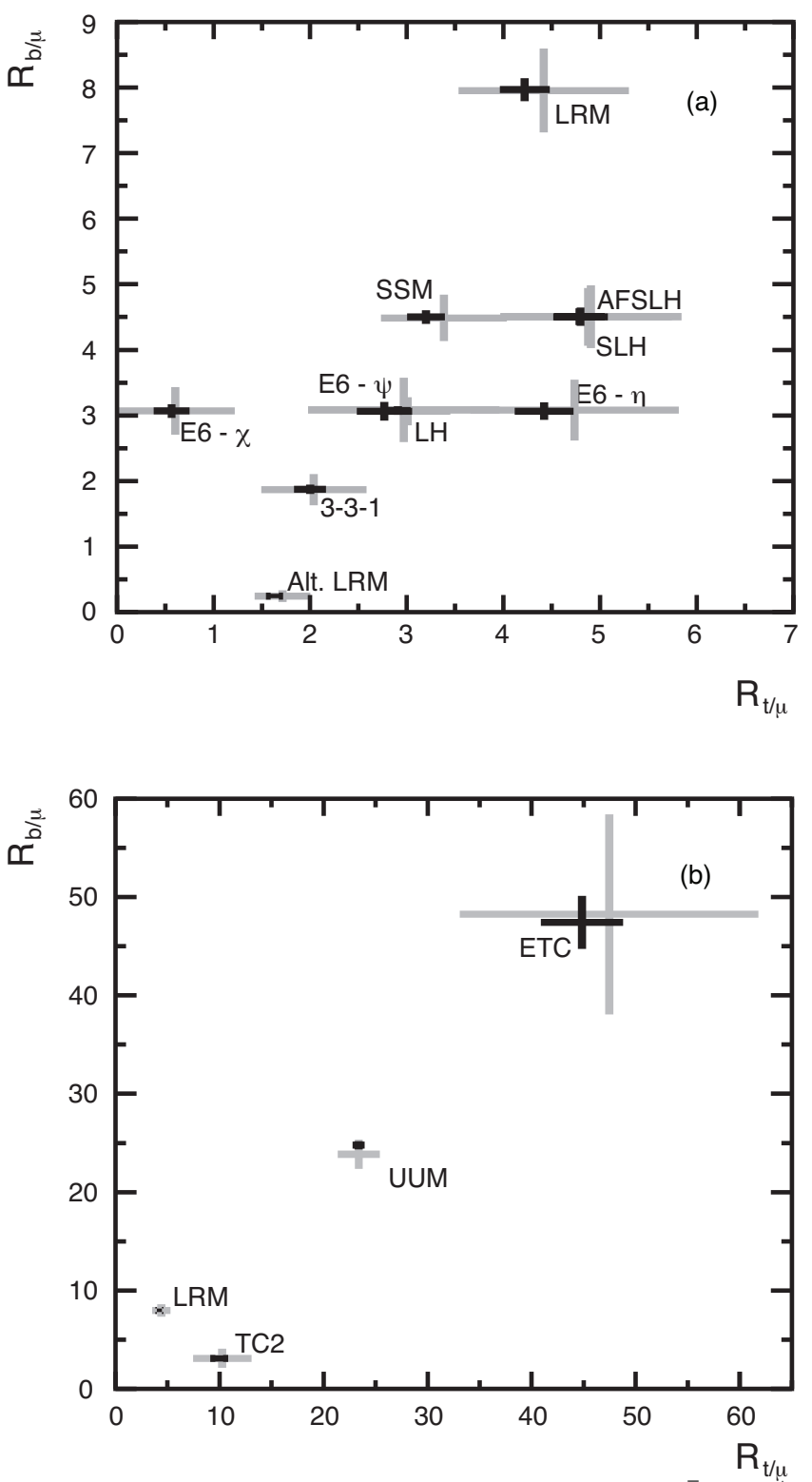

FIG. 4: Measurements of the ratio of $t \bar{t}$ and $b \bar{b}$ to $\mu^{+} \mu^{-}$ cross sections within $2.5 \Gamma$ of $M_{Z^{\prime}}$. Black bars correspond to expected $1 \sigma$ statistical uncertainties for $M_{Z^{\prime}}=1.5 \mathrm{TeV}$ and grey bars to $M_{Z^{\prime}}=2.5 \mathrm{TeV}$. (b) Expands the scales to include models with generation and family dependent couplings.

measurements. The experimentalists can try to disentangle resolution effects from the underlying cross section to give the "theoretical" cross section or they can compare to Monte Carlo simulations that include detector resolution. In any case, the sensitivity of measurements for narrow models to detector resolution will lead to systematic uncertainties in the measurements that need to be taken into account.

For some models, including models with non-universal couplings, the $E_{6}$ model, and the LR Symmetric models, the predictions for $R_{b / \mu}$ and $R_{t / \mu}$ are dependent on 

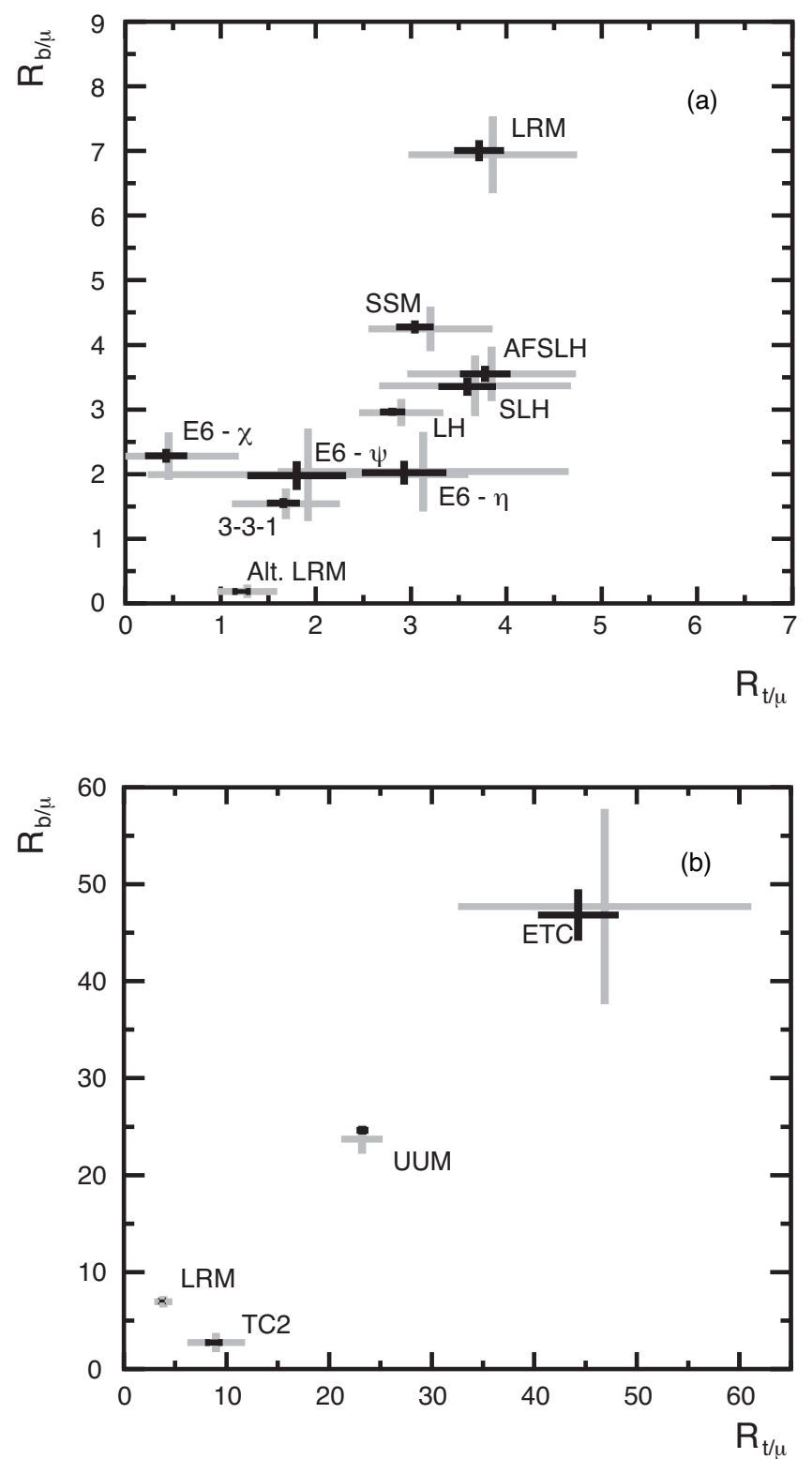

FIG. 5: Measurements of the ratio of $t \bar{t}$ and $b \bar{b}$ to $\mu^{+} \mu^{-}$cross sections within $2.5 \Gamma$ of $M_{Z^{\prime}}$ including detector resolution of $5 \%$ for $b$ and $t$ final states, and $3 \%$ for muons. The labelling is as in Fig. 4

the mixing angle between subgroups in the model. This is illustrated in Fig. 6 which plots the $R_{t / \mu}-R_{b / \mu}$ ratios while varying the mixing parameters. To obtain these curves, a $Z^{\text {/prime }}$ mass of $1.5 \mathrm{TeV}$ was used, although the results are not very sensitive to $M_{Z / \text { prime }}$, and the following ranges for mixing angles were used: for the LR Symmetric model, both with standard and alternate isospin assignments, the mixing parameter is constrained by $0.55 \leq\left(g_{R} / g_{L}\right) \leq 1$ [92]; for the UUM, $\phi$ is constrained by $0.22 \leq \sin \phi \leq 0.99$ [93]; and for the remaining models no specific limits could be found in the literature that were not directly tied to the mass of the $Z^{\prime}$. Depending on the mixing parameter, the predic-

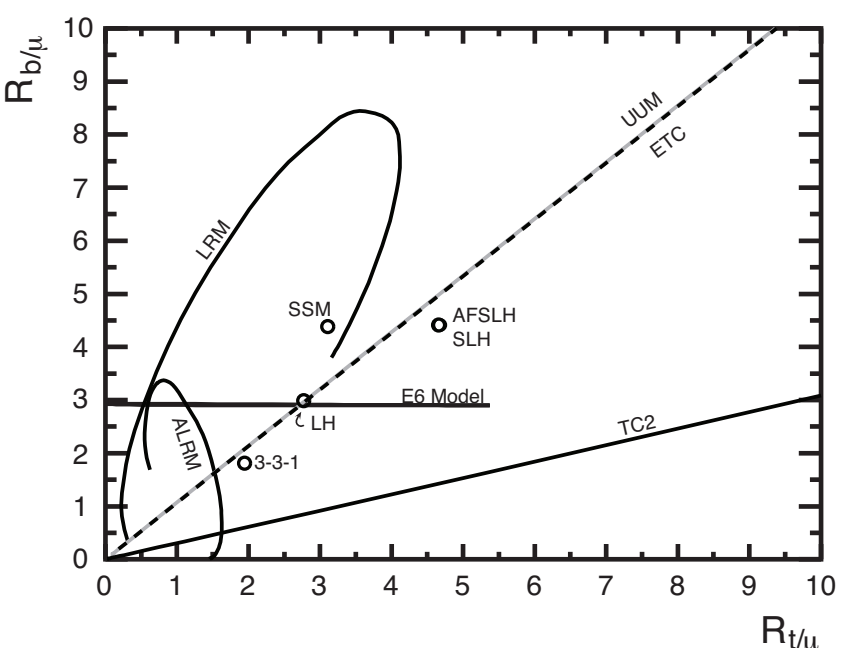

FIG. 6: $R_{b / \mu}$ vs $R_{t / \mu}$ spanning the model parameter range as given in the text, for the models labelled in the figure.

tions for some models overlap in the $R_{t / \mu}-R_{b / \mu}$ space as shown in Fig. 6. Consequently, other measurements will be needed to distinguish between models for these values of the model parameters.

It is important to note that the region of overlap in the $R_{t / \mu}-R_{b / \mu}$ plane between models with and without universal couplings occurs for parameter values that are not of particular interest. In the case of the UUM model, the overlap occurs for parameter values where leptons have preferential couplings and in the case of the ETC and TC2 models the overlap occurs when the first two generations of fermions have preferential couplings. Since these models are constructed such that the top quark plays a role in EWSB, one would not expect their mixing angles to take values in the overlap region.

A final observation is that the UUM and ETC models are indistinguishable using measurements of $R_{t / \mu}$ and $R_{b / \mu}$ for any value of mixing parameter. However, in this case, the ratio of tau to muon events at the LHC will discriminate generation dependent couplings. This is examined in the next subsection.

\section{Extracting Mixing Parameters Using $R_{\tau / \mu}$}

Measuring generation universality will be an important step in distinguishing or ruling out TC2 and ETC type models. The simplest and cleanest way to measure the level of universality is to determine the ratio of the $Z^{\prime}$ decays to $\tau$-leptons and to muons or electrons which can be found by measuring $\sigma\left(p p \rightarrow Z^{\prime} \rightarrow \tau^{+} \tau^{-}\right) / \sigma(p p \rightarrow$ $\left.Z^{\prime} \rightarrow \mu^{+} \mu^{-}\right)$.

We also considered a measurement of the ratio of $t$ - to $c$-quark cross sections but found that such a measurement does not appear promising due to low tagging efficiencies of the charm quark, and indistinguishability of the charm quark from the light jet backgrounds.

We therefore restrict ourselves to measurement of the 


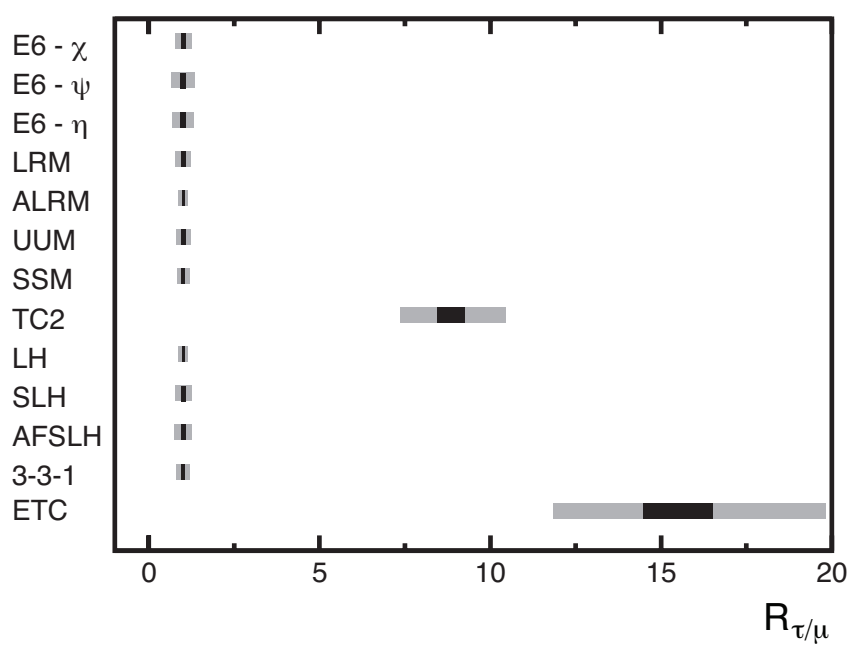

FIG. 7: $R_{\tau / \mu}$ for models with universal and non-universal fermion couplings. The ratios are obtained for tau and muon events satisfying $\left|M_{f \bar{f}}-M_{Z^{\prime}}\right|<2.5 \Gamma_{Z^{\prime}}$, along with the other cuts described in the text. The dark bars are for $M_{Z^{\prime}}=$ 1.5 TeV and the grey bars for $M_{Z^{\prime}}=2.5 \mathrm{TeV}$ (for $\sin \phi_{T C 2}=$ 0.5 and $\sin \phi_{E T C}=0.9$ ).

ratio $R_{\tau / \mu}=\sigma\left(p p \rightarrow Z^{\prime} \rightarrow \tau^{+} \tau^{-}\right) / \sigma\left(p p \rightarrow Z^{\prime} \rightarrow\right.$ $\mu^{+} \mu^{-}$), shown in Fig. 7. To obtain these results we imposed a cut on the invariant mass of the final state fermions of $\left|M_{Z^{\prime}}-M_{f \bar{f}}\right|<2.5 \Gamma_{Z^{\prime}}$ in addition to imposing a requirement of $p_{T_{f}}>0.3 M_{Z^{\prime}}$ and $\left|\eta_{f}\right|<2.5$. These results and those shown in Fig. 8 only include the statistical errors for a semi-idealized detector with perfect energy resolution. We will comment on this below.

It is clear from Fig. 7 that models with generation universality will yield measurements of $R_{\tau / \mu} \simeq 1$ with reasonable precision. In contrast, models with generation dependent couplings show a large, measurable variation from unity. The dependence of $R_{\tau / \mu}$ on the mixing angle between the gauge groups of the theory are given by:

$$
\frac{\Gamma\left(Z_{E T C}^{\prime} \rightarrow \tau^{+} \tau^{-}\right)}{\Gamma\left(Z_{E T C}^{\prime} \rightarrow \mu^{+} \mu^{-}\right)} \propto \tan ^{4} \phi_{E T C}
$$

and

$$
\frac{\Gamma\left(Z_{T C 2}^{\prime} \rightarrow \tau^{+} \tau^{-}\right)}{\Gamma\left(Z_{T C 2}^{\prime} \rightarrow \mu^{+} \mu^{-}\right)} \propto \cot ^{4} \phi_{T C 2}
$$

Given the fundamental nature of the gauge group mixing angle, measuring its precise value would be vital input into constructing the Lagrangian of the underlying theory. In Fig. 8] we show how well such a measurement can be made for the TC2 and ETC models using $R_{\tau / \mu}$ assuming $M_{Z^{\prime}}=1.5 \mathrm{TeV}$ and $L=100 \mathrm{fb}^{-1}$ for the semiidealized detector. In these plots the $x$-axis corresponds to the assumed value of the mixing parameter and the $y$-axis corresponds to the measured value of the mixing parameter with the spread in the vertical direction corresponding to 1 - and $2-\sigma$ limits of $R_{\tau / \mu}$ for the input parameter value and measured value. The parameter range corresponds to the range where the $Z^{\prime}$ width is less than $10 \%$ of the $Z^{\prime}$ mass. As before, we include the backgrounds in estimating the statistical errors and impose the same kinematic cuts as before. These limits could be further constrained by including more observables, such as $R_{b / \mu}$ and $R_{t / \mu}$ into the fit.

Figure 7 indicates that, for typical parameter values, the non-universal models predict values for $R_{\tau / \mu}$ that are very distinct from the value of $R_{\tau / \mu} \simeq 1$ expected for models with generation universality. As pointed out, these results do not include finite detector resolution and in addition, measurements of $\tau^{+} \tau^{-}$final states have the additional complication of missing energy due to neutrinos in the $\tau$ decays. However, the non-universal $Z^{/ \text {prime }}$ s have large decay widths so that including the measurement resolution [76, 78, 79] will only have a small effect on the results for non-universal $Z^{/ \text {prime }}$ s. On the other hand, some of the generation universal $Z^{\text {prime }}$ models are relatively narrow so that the resonances will be smeared out in the $\tau^{+} \tau^{-}$final state and some care will have to be taken in choosing appropriate invariant mass windows. We note that both ATLAS 94 and CMS 95] have recently measured $\sigma\left(p p \rightarrow Z^{0}\right) \times \mathcal{B}\left(Z^{0} \rightarrow \tau^{+} \tau^{-}\right)$ with errors of roughly $20 \%$ and $10 \%$ respectively in the $\tau_{h} \tau_{\ell}$ modes which bodes well for a measurement of $R_{\tau / \mu}$.

We conclude that, at worse, a very crude measurement of $R_{\tau / \mu}$ could signal generation non-universality. The mixing parameter measurement capability shown in Fig. 8 is an idealization but, given the recent ATLAS and CMS measurements of $Z^{0} \rightarrow \tau^{+} \tau^{-}$, we are optimistic that a measurement will be possible to constrain the relevant mixing angle.

\section{Forward-Backward Asymmetry with Heavy Quark Final States}

The ability to identify $b$ - and $t$-quarks at the LHC offers the possibility of using forward-backward asymmetries $\left(A_{F B}\right)$ in heavy quark final states to assist in the determination of individual fermion couplings to a $Z^{\prime}$ [8]. As will be seen below, $A_{F B}$ has a different dependence on the $Z^{\prime}$-fermion couplings than the $Z^{\prime}$ production cross section and $Z^{\prime}$ width.

Generally, a forward event is defined by the decay angle of the outgoing fermion relative to the direction of the interacting quark in the Drell-Yan annihilation. For $p p$ collisions at the LHC, there is an ambiguity in determining the direction of the quark, where it is impossible to tell on an event-by-event basis whether the $Z^{\prime}$ is boosted in the direction of the quark or anti-quark. Because the momentum distributions are harder for valence quarks than for sea anti-quarks, this ambiguity can be resolved to a certain extent by assuming that the $Z^{\prime}$ boost direction is the same as the quark direction [96]. In the central region of $Z^{\prime}$ rapidity, the quark and anti-quark momenta are more evenly balanced, and the correct and incorrect assignments are canceled in an asymmetry measurement. 

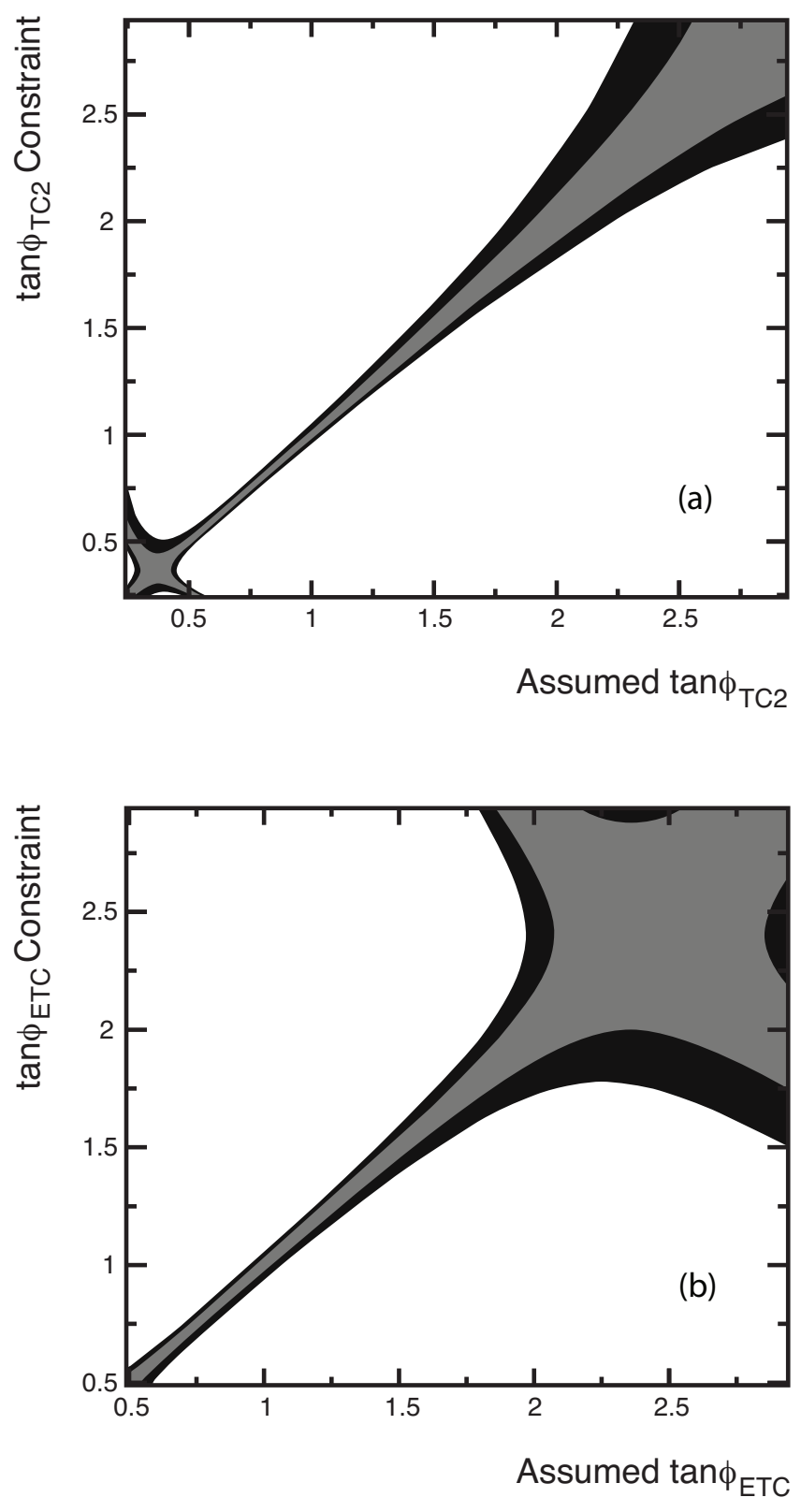

FIG. 8: LHC measurement capability of the mixing parameter using $R_{\tau / \mu}$ for (a) TC2 and (b) ETC. The horizontal axis corresponds to the input parameter value and the verticle axis corresponds to the extracted value with 1- (grey) and 2- (black) $\sigma$ limits. $M_{Z^{\prime}}=1.5 \mathrm{TeV}$ and an integrated luminosity of $100 \mathrm{fb}^{-1}$ was assumed in these plots with the following kinematic cuts; $|\eta|<2.5,\left|M_{l^{+} l^{-}}-M_{Z^{\prime}}\right|<2.5 \Gamma_{Z^{\prime}}$, $p_{T}<0.3 M_{Z^{\prime}}$.

In a recent paper [8], we suggested a simple method of performing the forward-backward asymmetry measurement by using the direct pseudorapidity measurements of the final state particles. This method differs from the traditional definition given in [91, 97, 98]. It can be shown that a "forward" event is one in which $\left|\eta_{f}\right|>\left|\eta_{\bar{f}}\right|$ in the lab frame, and vice-versa for a "backward" event, assuming that the $Z^{\prime}$ is boosted by the quark as in the traditional definition. Using these forms for forward and backward events, the forward-backward asymmetry of the signal is given by:

$$
\begin{aligned}
A_{F B} & =\frac{\int F(y)-B(y) d y}{\int F(y)+B(y) d y} \\
& \sim\left(\frac{L_{Q}^{2}-R_{Q}^{2}}{L_{Q}{ }^{2}+R_{Q}{ }^{2}}\right)\left(\frac{\sum_{q} G_{q}^{-}\left(L_{q}^{2}-R_{q}^{2}\right)}{\sum_{q} G_{q}^{+}\left(L_{q}^{2}+R_{q}^{2}\right)}\right)
\end{aligned}
$$

where $F(y)$ is the number of forward events and $B(y)$ is the number of backward events for a given $Z^{\prime}$ rapidity, $y$. The approximation is a representation of the on-peak contribution to the $A_{F B}$ that more clearly indicates the coupling dependencies. $L_{f}$ and $R_{f}$ are the left and right handed couplings of the $Z^{\prime}$ to the fermions, and $G_{q}^{ \pm}$are the integrated symmetric and anti-symmetric combinations of the parton distribution functions.

This method for finding the $A_{F B}$ has the advantage of being very straightforward and clean. It simply relies on counting events with $\left|\eta_{f}\right|>\left|\eta_{\bar{f}}\right|$ and $\left|\eta_{f}\right|<\left|\eta_{\bar{f}}\right|$. No calculation of the centre-of-mass scattering angle or $Z^{\prime}$ rapidity is required. In Fig. 9, we show the $A_{F B}$ distribution as a function of invariant mass of the $b \bar{b}$ and $t \bar{t}$ final states for several representative models with a $Z^{\prime}$ mass of $1.5 \mathrm{TeV}$. It should be noted that it is unlikely there will be sufficient statistics to make this measurement except on the $Z^{\text {/prime }}$ resonance.

As in all measurements involving third generation fermions, the challenge is extracting the events of interest from a large standard model background and accumulating sufficient statistics to make a meaningful measurement. The QCD backgrounds for these measurements are forward-backward symmetric at tree level, which should allow a heavy quark $A_{F B}$ to be sensitive to the presence of a $Z^{\prime}$. Rather than subtracting out the backgrounds we include the totals of signal plus backgrounds in the forward and backward regions. Since the backgrounds are symmetric, they do not contribute to the numerator, but do for the denominator. This has the effect of making the magnitude of the asymmetry smaller but also has the advantage of reducing the statistical errors. The statistical errors include contributions from both QCD heavy quark backgrounds and the reduced light dijet background.

Figure [10] shows the expected results for $A_{F B}$ in the $b \bar{b}$ and $t \bar{t}$ channels for several models assuming $M_{Z^{\prime}}=$ $1.5 \mathrm{TeV}, L=100 \mathrm{fb}^{-1}$, and the same kinematic cuts described previously, not including detector resolution effects. While large uncertainties are apparent for some models, a reasonable measurement can still be expected for most models, including the Left-Right Symmetric Model, the various Little Higgs models, and models with non-universal couplings. Table Igives the corresponding 

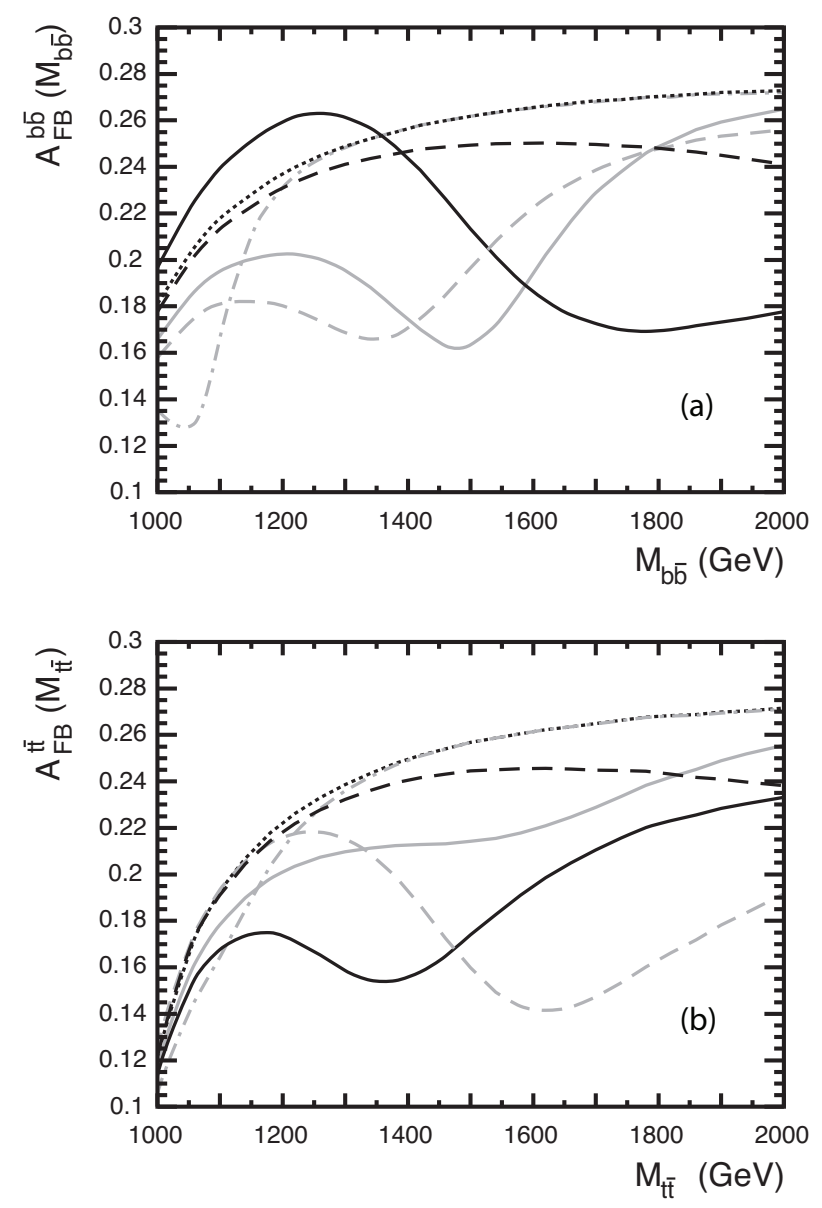

FIG. 9: (a) $A_{F B}^{b \bar{b}}$ and (b) $A_{F B}^{t \bar{t}}$ of the $Z^{\prime}$ signal as a function of invariant mass for a $Z^{\prime}$ with $M_{Z^{\prime}}=1.5 \mathrm{TeV}$. Cuts employed include $p_{T}>0.3 M_{Z^{\prime}}$ and $|\eta|<2.5$. These figures do not include backgrounds, and only examine the asymmetry distribution of $p p \rightarrow \gamma / Z^{0} / Z^{\prime} \rightarrow q \bar{q}$. Models follow the same format as Fig. 2. ETC (dashed, dark), TC2 (solid, grey), UUM (dotted, dark), AFSLH (dashed, grey), SLH (solid, dark), SSM (dotted, grey) and LH (dot-dash, grey). The results are for the "ideal" case that doesn't take into account detector resolution.

numerical values for $A_{F B}^{t \bar{t}, b \bar{b}}$ and the statistical uncertainties that can be expected for $100 \mathrm{fb}^{-1}$ integrated luminosity.

Fig. 11 shows similar results but this time including the detector resolution as described earlier. In this figure we assumed an integrated luminosity of $300 \mathrm{fb}^{-1}$ to improve the statistics. Taking into account detector resolution both reduces the statistics and shifts the observed values for $A_{F B}$. While $A_{F B}$ still has some resolving power with $b \bar{b}$ final states it is not clear how useful the $t \bar{t}$ final states will be, due to low statistics. It is possible that if some effort were made to disentangle detector resolution from the underlying cross section these results could be improved. Nevertheless, we expect that $A_{F B}$ measurements would help constrain the $Z^{\prime}$-fermion couplings as part of a global fit.

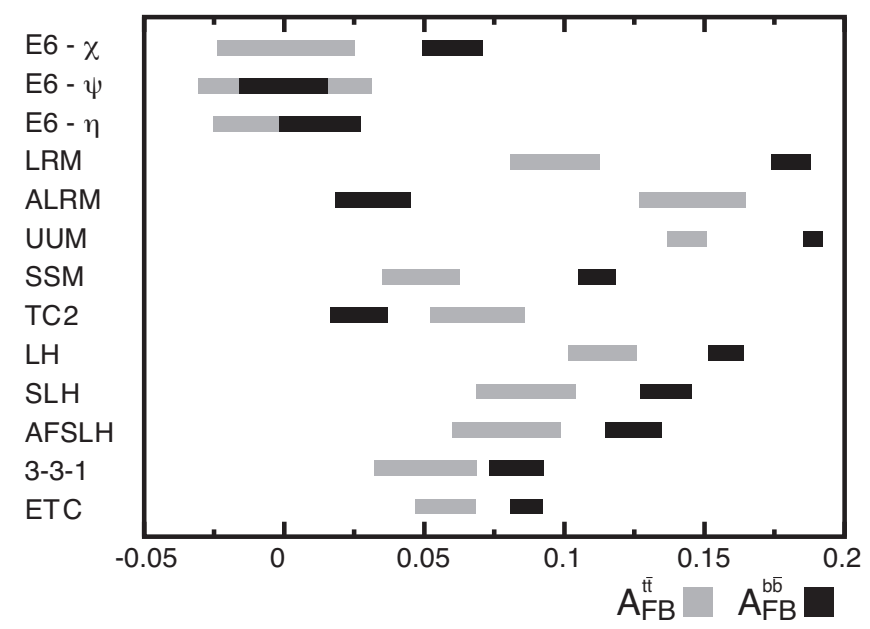

FIG. 10: $A_{F B}^{b \bar{b}}$ (black bars) and $A_{F B}^{t \bar{t}}$ (grey bars) for a $Z^{\prime}$ with a mass of $1.5 \mathrm{TeV}$. Statistical errors include contributions from QCD backgrounds and light dijets assuming $100 \mathrm{fb}^{-1}$ luminosity.

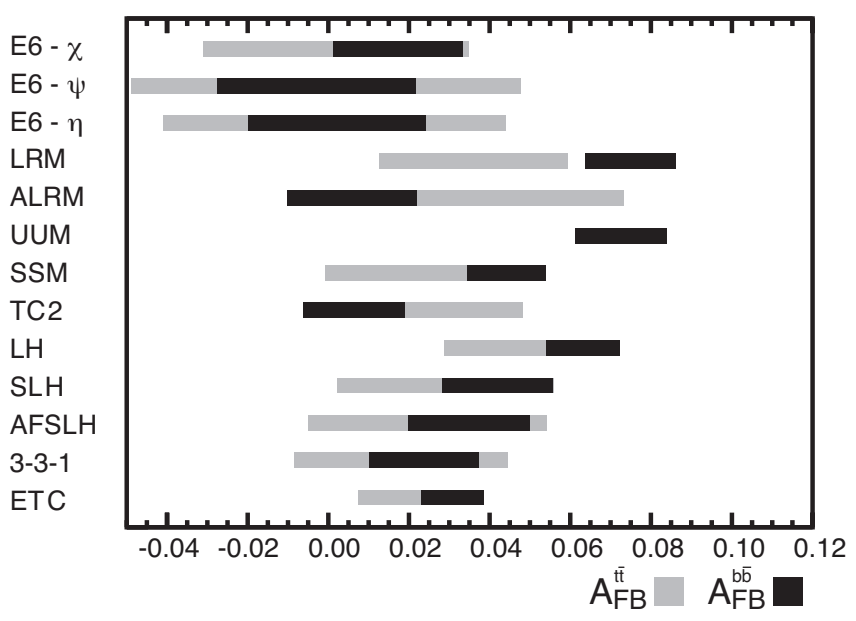

FIG. 11: $A_{F B}^{b \bar{b}}$ (black bars) and $A_{F B}^{t \bar{t}}$ (grey bars) including detector resolution as described in the text. Statistical errors include contributions from QCD backgrounds and light dijets assuming $300 \mathrm{fb}^{-1}$ luminosity.

\section{SUMMARY}

Extra neutral gauge bosons are a hallmark of many models of physics beyond the standard model and may be discovered early in the LHC program. In this paper, we expanded upon our previous phenomenological study by focusing on measurements using third generation fermions and exploring models with non-universal fermion- $Z^{\prime}$ couplings. We first gave an update of $Z^{\prime}$ discovery limits including non-universal coupling models and the LHC energy and planned luminosity for the 2010-2011 run. We found that this run will roughly double the mass reach of the Fermilab Tevatron.

The main focus of this paper was to explore the useful- 
TABLE II: $A_{F B}$ values for $b$ - and $t$-quark final states with corresponding statistical uncertainties, assuming $M_{Z^{\prime}}=1.5$ TeV and $L=100 \mathrm{fb}^{-1}$. Cuts include $p_{T}>0.3 M_{Z^{\prime}} \mathrm{GeV},\left|\eta_{t, b}\right|<2.5$, within $\left|\Delta M_{q \bar{q}}-M_{Z^{\prime}}\right|<2.5 \Gamma_{Z^{\prime}}$. The first set of data, labelled as "ideal detector", do not include detector resolution smearing for the final state fermions, while the second set of data does include the effects of detector resolution.

\begin{tabular}{|c|c|c|c|c|c|c|c|c|}
\hline Model & $A_{F B}^{t} \pm$ & $\delta A_{F B}^{t}$ & $A_{F B}^{b} \pm$ & $\delta A_{F B}^{b}$ & $A_{F B}^{t} \pm$ & $\delta A_{F B}^{t}$ & $A_{F B}^{b} \pm$ & $\delta A_{F B}^{b}$ \\
\hline & \multicolumn{4}{|c|}{ ideal detector } & \multicolumn{4}{|c|}{ including detector resolution } \\
\hline$E_{6} \chi$ & $0.00 \pm$ & 0.02 & $0.060 \pm$ & 0.011 & $0.00 \pm$ & 0.03 & $0.017 \pm$ & 0.016 \\
\hline$E_{6} \psi$ & $0.00 \pm$ & 0.03 & $0.000 \pm$ & 0.016 & $0.00 \pm$ & 0.05 & $0.00 \pm$ & 0.02 \\
\hline$E_{6} \eta$ & $0.00 \pm$ & 0.03 & $0.013 \pm$ & 0.014 & $0.00 \pm$ & 0.04 & $0.00 \pm$ & 0.02 \\
\hline LRM & $0.097 \pm$ & 0.016 & $0.181 \pm$ & 0.007 & $0.04 \pm$ & 0.02 & $0.075 \pm$ & 0.011 \\
\hline ALRM & $0.146 \pm$ & 0.019 & $0.032 \pm$ & 0.013 & $0.04 \pm$ & 0.03 & $0.006 \pm$ & 0.016 \\
\hline UUM & $0.144 \pm$ & 0.007 & $0.189 \pm$ & 0.004 & $0.073 \pm$ & 0.011 & $0.094 \pm$ & 0.006 \\
\hline SSM & $0.049 \pm$ & 0.014 & $0.112 \pm$ & 0.007 & $0.02 \pm$ & 0.02 & $0.044 \pm$ & 0.010 \\
\hline $\mathrm{TC} 2$ & $0.069 \pm$ & 0.017 & $0.027 \pm$ & 0.010 & $0.02 \pm$ & 0.02 & $0.01 \pm$ & 0.01 \\
\hline $\mathrm{LH}$ & $0.114 \pm$ & 0.012 & $0.158 \pm$ & 0.006 & $0.047 \pm$ & 0.018 & $0.063 \pm$ & 0.009 \\
\hline $\mathrm{SLH}$ & $0.086 \pm$ & 0.018 & $0.136 \pm$ & 0.009 & $0.03 \pm$ & 0.03 & $0.042 \pm$ & 0.014 \\
\hline AFSLH & $0.079 \pm$ & 0.019 & $0.124 \pm$ & 0.010 & $0.02 \pm$ & 0.03 & $0.035 \pm$ & 0.015 \\
\hline $3-3-1(2 \mathrm{U} 1 \mathrm{D})$ & $0.050 \pm$ & 0.018 & $0.083 \pm$ & 0.010 & $0.02 \pm$ & 0.03 & $0.023 \pm$ & 0.014 \\
\hline ETC & $0.058 \pm$ & 0.011 & $0.086 \pm$ & 0.006 & $0.022 \pm$ & 0.015 & $0.031 \pm$ & 0.008 \\
\hline
\end{tabular}

ness of third generation fermions in studying extra neutral gauge bosons. We found that it should be possible to measure the decays of a moderately heavy $Z^{\prime}$ to third generation quarks, depending on the capability of the experiments to reject against the light jet background. Such measurements would prove to be very effective at distinguishing between different models of $Z^{\prime}$. The measurement of the ratio of $\tau$ to $\mu$ decays of the $Z^{\prime}$ should be very effective in testing generation universality of the $Z^{\prime}$. We also studied using the forward backward asymmetry of $b$ - and $t$-quarks from $Z^{\prime}$ decays to distinguish models. For a $Z^{\prime}$ of moderate mass these measurements could help distinguish between different models. More importantly, they can contribute valuable input for measuring $Z^{\prime}$-fermion couplings, and should be an integral piece of a global fit with this goal.

An important source of uncertainty in these measurements is the degradation of the heavy fermion final state signals due to detector resolution. It appears to be manageable for $b \bar{b}$ and $t \bar{t}$ final states for broad $Z^{/ \text {prime }}$ resonances such as in the TC2 and ETC models, but becomes increasingly important for narrow resonances such as the
E6 models. Now that ATLAS and CMS have demonstrated the ability to measure $\sigma\left(p p \rightarrow Z^{0}\right) \times \mathcal{B}\left(Z^{0} \rightarrow\right.$ $\tau^{+} \tau^{-}$) we are optimistic that $R_{\tau / \mu}$ can be measured well enough to distinguish between typical generation nonuniversal models and generation universal models. Finally, we found that detector resolution effects can degrade $A_{F B}$ measurements quite significantly, in particular for $t \bar{t}$ final states. We believe that measurements using 3rd generation fermions look promising but clearly, a more careful detector level study is needed to properly take into account detector resolution and other complications of a real detector.

\section{Acknowledgments}

This research was supported in part by the Natural Sciences and Engineering Research Council of Canada. The authors thank Dag Gillberg, David Morrissey, Dugan O'Neil, Oliver Stelzer-Chilton, and Isabel Trigger for helpful discussions.

[1] P. Langacker, Rev. Mod. Phys. 81, 1199 (2008) arXiv:0801.1345 [hep-ph]].

[2] A. Leike, Phys. Rept. 317, 143 arXiv:hep-ph/9805494. 
[3] M. Cvetic and S. Godfrey, arXiv:hep-ph/9504216

[4] J. L. Hewett and T. G. Rizzo, Phys. Rept. 183, 193 (1989).

[5] T. G. Rizzo, arXiv:hep-ph/0610104.

[6] F. Petriello and S. Quackenbush, Phys. Rev. D 77, 115004 (2008) arXiv:0801.4389 [hep-ph]].

[7] S. Godfrey and T. A. W. Martin, Phys. Rev. Lett. 101, 151803 (2008) arXiv:0807.1080 [hep-ph]].

[8] R. Diener, S. Godfrey and T. A. W. Martin, Phys. Rev. D 80, 075014 (2009) arXiv:0909.2022 [hep-ph]].

[9] R. Diener, S. Godfrey and T. A. W. Martin, arXiv:0910.1334 [hep-ph].

[10] Y. Li, F. Petriello and S. Quackenbush, Phys. Rev. D 80, 055018 (2009) arXiv:0906.4132 [hep-ph]].

[11] F. del Aguila, J. A. Aguilar-Saavedra, M. Moretti, F. Piccinini, R. Pittau and M. Treccani, Phys. Lett. B 685, 302 (2010) arXiv:0912.3799 [hep-ph]].

[12] V. Barger, P. Langacker and H. S. Lee, Phys. Rev. Lett. 103, 251802 (2009) arXiv:0909.2641 [hep-ph]].

[13] P. Osland, A. A. Pankov, A. V. Tsytrinov and N. Paver, Phys. Rev. D 79, 115021 (2009) arXiv:0904.4857 [hep$\mathrm{ph}]$.

[14] T. G. Rizzo, JHEP 0908, 082 (2009) arXiv:0904.2534 [hep-ph]].

[15] G. Aad et al. [ATLAS Collaboration], JINST 3, S08003 (2008).

[16] G. L. Bayatian et al. [CMS Collaboration], J. Phys. G 34 (2007) 995.

[17] H. Georgi, E. E. Jenkins and E. H. Simmons, Nucl. Phys. B 331, 541 (1990).

[18] R. S. Chivukula, E. H. Simmons and J. Terning, Phys. Lett. B 346, 284 (1995) arXiv:hep-ph/9412309. topflavour models 21 23]

[19] R. S. Chivukula and E. H. Simmons, Phys. Rev. D 66, 015006 (2002) arXiv:hep-ph/0205064.

[20] W. A. Bardeen, C. T. Hill and M. Lindner, Phys. Rev. D 41, 1647 (1990).

[21] E. Malkawi, T. Tait and C. P. Yuan, Phys. Lett. B 385, 304 (1996) arXiv:hep-ph/9603349.

[22] D. J. Muller and S. Nandi, arXiv:hep-ph/9610404

[23] H. J. He, T. Tait and C. P. Yuan, Phys. Rev. D 62, 011702 (2000) arXiv:hep-ph/9911266.

[24] R. S. Chivukula, E. H. Simmons and J. Terning, Phys. Lett. B 331, 383 (1994) arXiv:hep-ph/9404209].

[25] R. S. Chivukula, E. H. Simmons and J. Terning, Phys. Rev. D 53, 5258 (1996) arXiv:hep-ph/9506427.

[26] E. H. Simmons, Phys. Rev. D 55, 5494 (1997) arXiv:hep-ph/9612402.

[27] C. T. Hill, Phys. Lett. B 345, 483 (1995) arXiv:hep-ph/9411426.

[28] K. D. Lane and E. Eichten, Phys. Lett. B 352, 382 (1995) arXiv:hep-ph/9503433.

[29] K. D. Lane, Phys. Rev. D 54, 2204 (1996) arXiv:hep-ph/9602221.

[30] K. D. Lane, Phys. Lett. B 433, 96 (1998) arXiv:hep-ph/9805254.

[31] M. B. Popovic and E. H. Simmons, Phys. Rev. D 58, 095007 (1998) arXiv:hep-ph/9806287.

[32] T. G. Rizzo, arXiv:hep-ph/9306312.

[33] T. Han, H. E. Logan and L. T. Wang, JHEP 0601, 099 (2006) arXiv:hep-ph/0506313.

[34] K. R. Lynch, E. H. Simmons, M. Narain and S. Mrenna, Phys. Rev. D 63, 035006 (2001) arXiv:hep-ph/0007286].

[35] R. M. Harris, C. T. Hill and S. J. Parke,
arXiv:hep-ph/9911288

[36] U. Baur and L. H. Orr, Phys. Rev. D 77, 114001 (2008) arXiv:0803.1160 [hep-ph]].

[37] R. Frederix and F. Maltoni, JHEP 0901, 047 (2009) arXiv:0712.2355 [hep-ph]].

[38] P. K. Mohapatra, Mod. Phys. Lett. A 8, 771 (1993) arXiv:hep-ph/9212259.

[39] K. Agashe et al., Phys. Rev. D 76, 115015 (2007) arXiv:0709.0007] [hep-ph]].

[40] See also: S. Chekanov and J. Proudfoot, Phys. Rev. D 81, 114038 (2010) arXiv:1002.3982 [hep-ph]]; A. Katz, M. Son and B. Tweedie, arXiv:1010.5253 [hep-ph].

[41] N. Arkani-Hamed, A. G. Cohen, E. Katz and A. E. Nelson, JHEP 0207, 034 (2002) arXiv:hep-ph/0206021.

[42] M. Schmaltz, JHEP 0408, $056 \quad$ (2004) arXiv:hep-ph/0407143.

[43] D. E. Kaplan and M. Schmaltz, JHEP 0310, 039 (2003) arXiv:hep-ph/0302049.

[44] F. Pisano and V. Pleitez, Phys. Rev. D 46, 410 (1992) arXiv:hep-ph/9206242

[45] C. T. Hill, Phys. Lett. B 266, 419 (1991).

[46] C. T. Hill and E. H. Simmons, Phys. Rept. 381, 235 (2003) [Erratum-ibid. 390, 553 (2004)] arXiv:hep-ph/0203079.

[47] S. Godfrey, J. L. Hewett and T. G. Rizzo, Phys. Rev. D 37, 643 (1988).

[48] M. Dittmar, A. S. Nicollerat and A. Djouadi, Phys. Lett. B 583, 111 (2004) arXiv:hep-ph/0307020.

[49] C. Amsler et al. [Particle Data Group], Phys. Lett. B 667, 1 (2008).

[50] J. Pumplin, D. R. Stump, J. Huston, H. L. Lai, P. M. Nadolsky and W. K. Tung, JHEP 0207, 012 (2002) arXiv:hep-ph/0201195.

[51] J. Kubar-Andre and F. E. Paige, Phys. Rev. D 19, 221 (1979).

[52] K. Melnikov and F. Petriello, Phys. Rev. D 74, 114017 (2006) arXiv:hep-ph/0609070.

[53] C. Anastasiou, L. J. Dixon, K. Melnikov and F. Petriello, Phys. Rev. D 69, 094008 (2004) arXiv:hep-ph/0312266.

[54] A. L. Kataev, Phys. Lett. B 287, 209 (1992).

[55] S. G. Gorishnii, A. L. Kataev and S. A. Larin, Phys. Lett. B 212, 238 (1988).

[56] G. D'Agostini, W. de Boer and G. Grindhammer, Phys. Lett. B 229, 160 (1989).

[57] U. Baur, O. Brein, W. Hollik, C. Schappacher and D. Wackeroth, Phys. Rev. D 65, 033007 (2002) arXiv:hep-ph/0108274.

[58] U. Baur, S. Keller and W. K. Sakumoto, Phys. Rev. D 57, 199 (1998) arXiv:hep-ph/9707301.

[59] U. Baur and D. Wackeroth, Nucl. Phys. Proc. Suppl. 116, 159 (2003) arXiv:hep-ph/0211089.

[60] W. Kilian, T. Ohl and J. Reuter, arXiv:0708.4233 [hep$\mathrm{ph}$.

[61] T. Ohl, Presented at LoopFest V: Radiative Corrections for the International Linear Collider: Multi-loops and Multi-legs, SLAC, Menlo Park, California, 19-21 Jun 2006

[62] W. Kilian, "WHIZARD 1.0: A generic Monte-Carlo integration and event generation package for multi-particle processes." LC-TOOL-2001-039

[63] F. Maltoni and T. Stelzer, JHEP 0302, 027 (2003) arXiv:hep-ph/0208156.

[64] V. D. Barger and R. J. N. Phillips, REDWOOD CITY, USA: ADDISON-WESLEY (1987) 592 P. (FRON- 
TIERS IN PHYSICS, 71)

[65] D. E. Kaplan, K. Rehermann, M. D. Schwartz and B. Tweedie, Phys. Rev. Lett. 101, 142001 (2008) arXiv:0806.0848 [hep-ph]].

[66] J. Thaler and L. T. Wang, JHEP 0807, 092 (2008) arXiv:0806.0023 [hep-ph]].

[67] U. Baur and L. H. Orr, Phys. Rev. D 76, 094012 (2007) arXiv:0707.2066 [hep-ph]].

[68] O. Kortner, arXiv:0707.0905 [hep-ex].

[69] D. L. Rainwater, D. Zeppenfeld and K. Hagiwara, Phys. Rev. D 59, 014037 (1999) arXiv:hep-ph/9808468.

[70] B. Holdom, Phys. Lett. B 666, 77 (2008) arXiv:0805.1965 [hep-ph]].

[71] V. Barger, T. Han and D. G. E. Walker, Phys. Rev. Lett. 100, 031801 (2008) arXiv:hep-ph/0612016.

[72] W. Wagner, Rept. Prog. Phys. 68, 2409 (2005) arXiv:hep-ph/0507207.

[73] L. G. Almeida, S. J. Lee, G. Perez, G. Sterman and I. Sung, Phys. Rev. D 82, 054034 (2010) arXiv:1006.2035 [hep-ph]].

[74] K. Agashe, A. Belyaev, T. Krupovnickas, G. Perez and J. Virzi, Phys. Rev. D 77, 015003 (2008) arXiv:hep-ph/0612015.

[75] The ATLAS Collaboration, ATLAS-CONF-2010-054 (2010).

[76] G. Aad et al. [The ATLAS Collaboration], arXiv:0901.0512 [hep-ex].

[77] The ATLAS Collaboration, ATL-PHYS-2010-008 (2010). See also ATL-PHYS-PUB-2009-081 (2009).

[78] K. J. C. Leney [ATLAS Collaboration], arXiv:0810.3144 [hep-ex].

[79] A. Elagin, P. Murat, A. Pranko and A. Safonov, arXiv:1012.4686 [hep-ex].

[80] S. Godfrey, Phys. Rev. D 51, 1402 (1995) arXiv:hep-ph/9411237.

[81] J. Kang and P. Langacker, Phys. Rev. D 71, 035014 (2005) arXiv:hep-ph/0412190.

[82] S. Godfrey, in Proc. of the APS/DPF/DPB Summer Study on the Future of Particle Physics (Snowmass 2001) ed. N. Graf, In the Proceedings of APS / DPF / DPB
Summer Study on the Future of Particle Physics (Snowmass 2001), Snowmass, Colorado, 30 Jun - 21 Jul 2001, pp P344 arXiv:hep-ph/0201093.

[83] S. Capstick and S. Godfrey, Phys. Rev. D 37, 2466 (1988).

[84] E. N. Thompson [ATLAS Collaboration], arXiv:0810.2928 [hep-ex].

[85] "ATLAS: Detector and physics performance technical design report. Vol. 1," CERN-LHCC-99-14

"ATLAS detector and physics performance. Technical design report. Vol. 2," CERN-LHCC-99-15

[86] T. Aaltonen et al. [CDF Collaboration], Phys. Rev. Lett. 102, 031801 (2009) arXiv:0810.2059 [hep-ex]].

[87] T. Aaltonen et al. [CDF Collaboration], Phys. Rev. Lett. 102, 091805 (2009).

[88] J. Erler, P. Langacker, S. Munir and E. R. Pena, JHEP 0908, 017 (2009) arXiv:0906.2435 [hep-ph]].

[89] F. del Aguila, J. de Blas and M. Perez-Victoria, arXiv:1005.3998 [hep-ph]].

[90] V. D. Barger, W. Y. Keung and E. Ma, Phys. Rev. D 22, 727 (1980). R. W. Robinett and J. L. Rosner, Phys. Rev. D 25, 3036 (1982) [Erratum-ibid. D 27, 679 (1983)]; See also Ref. [1 [5] and references therein.

[91] P. Langacker, R. W. Robinett and J. L. Rosner, Phys. Rev. D 30, 1470 (1984).

[92] D. Chang, R. N. Mohapatra and M. K. Parida, Phys. Rev. D 30, 1052 (1984).

[93] V. D. Barger and T. Rizzo, Phys. Rev. D 41, 946 (1990).

[94] The ATLAS Collaboration, ATL-PHYS-2011-010 (2011).

[95] S. Chatrchyan et al. [CMS Collaboration], arXiv:1104.1617 [hep-ex].

[96] M. Dittmar, Phys. Rev. D 55, 161 (1997).

[97] J. D. Anderson, M. H. Austern and R. N. Cahn, Phys. Rev. D 46, 290 (1992);

[98] F. del Aguila, M. Cvetic and P. Langacker, Phys. Rev. D 48, 969 (1993) arXiv:hep-ph/9303299; M. Cvetic and P. Langacker, Phys. Rev. D 46, 4943 (1992) [Erratumibid. D 48, 4484 (1993)] arXiv:hep-ph/9207216]. 